CHINESE JOURNAL OF PHYSICS

ISSN: 0577-9073; Impact factor = 2.638; Publisher - Elsevier

Accepted August 16 ${ }^{\text {th }} 2020$

\title{
Electro-osmotic Nanofluid Flow in a Curved Microchannel
}

\author{
V. K. Narla ${ }^{\mathrm{a}}$, Dharmendra Tripathi ${ }^{\mathrm{b}, *}$, O. Anwar Bég ${ }^{\mathrm{c}}$ \\ ${ }^{a}$ Department of Mathematics, School of Technology, GITAM, Hyderabad-502329, India \\ ${ }^{b}$ Department of Sciences and Humanities, National Institute of Technology, \\ Uttarakhand-2461\%4, India \\ ${ }^{c}$ Aeronautical and Mechanical Engineering, University of Salford, Manchester, M54WT, \\ $U K$
}

\begin{abstract}
Biological mechanisms offer significant improvement in the efficiency of next generation energy systems. Motivated by new developments in distensible pumping systems, ionic electro-kinetic manipulation and nanoscale liquids ("nanofluids"), in the present study a mathematical model is developed to simulate the entropy generation and electro-osmotic transport of nanofluids in a curved deformable microchannel driven by peristaltic transport. Both thermal and species (nano-particle) buoyancy effects are included and Soret and Dufour cross-diffusion effects. The appropriate conservation equations are normalized with scaled variables and the resulting dimensionless nonlinear boundary value problem is solved in a transformed coordinate system. Simplification of the mathematics is achieved via lubrication approximations and low zeta potential (Debye Hückel linearization). The effects of various parameters, i.e. electro-osmotic velocity, EDL (electrical double layer) thickness and zeta potential ratio on velocity profile and temperature profiles are computed. The effects of Brinkman number (viscous heating parameter) and Joule (electrical field heating) parameter on nano-particle concentration profiles are also simulated. The micro-channel curvature effects on the nanofluid flow characteristics and thermal characteristics are also computed. Furthermore, streamline patterns, temperature contours, nano-particles concentration contours and entropy generation rate contours are plotted for various
\end{abstract}

\footnotetext{
*Dharmendra Tripathi

Email addresses: vknarla@gmail.com (V. K. Narla), dtripathi@nituk.ac.in (Dharmendra Tripathi), gortoab@gmail.com (O. Anwar Bég)
} 
curvature parameters. Results indicate that the curvature of the channel and electro-osmotic body force influence strongly the sources of entropy generation rate. The study finds applications in bio-inspired electro-osmotic nanofluid pumping in microscale energy applications.

Keywords: Electro-osmosis, Peristalsis, Curvature effects, Nanofluids, Entropy, Hybrid deformable microchannels

\section{Introduction}

Transport phenomena in micro-channels feature in an extensive range of modern energy and chemical engineering applications including biofuel emulsification (Belkadi et al. [1]), heat exchangers (Qasem et al. [2]), thermal power technology (Norouzi et al. [3]), solar collectors (Deng et al. [4]), polymer processing (Norouzi et al. [5]), condenser design (Fronk and Zada [6]) and rocket propulsion (Bég et al. [7]). Other applications include naphtha reforming and sustainable fuel cell systems. The development of microscale heat transfer devices based on micro-channels are focused on offering a high heat transfer coefficient in a compact size. Microchannels also provide an enhanced surface-to-volume ratio, which achieves elevated heat and mass transfer rates and greater economic benefits. The fluid dynamics of microchannels has been lucidly studied by (Hestroni et al. [8]). Medical applications of micro-channels have also emerged in recent years and have embraced medical diagnosis, biomolecular manipulation (Chou et al. [9]), down-scaled bioreactors (Chen et al. [10]) and microfluidic devices for separating the subpopulations of red blood cells in malaria, sickle cell and other hematologic diseases (Ahmed et al. [11]). Heat transfer processes in micro-channels, viscous dissipation effects can be significant. This relates to the irreversible process via which work done by a fluid on adjacent layers due to the action of shear forces is transformed into heat, i.e. mechanical energy is destroyed. Inclusion of viscous heating in mathematical models has been shown to produce results which deviate significantly for non-dissipative systems. An extensive number of theoretical and computational studies have been communicated on thermal transport in micro-channels with viscous heating. These include the studies by Tunc and Bayazitoglu [12] (on micro-tubes) and Ragueb and Mansouri [13] (on elliptical cross-section micro-ducts using a dynamic Alternating Direction Implicit method). Morini and Spiga [14] analyzed the impact of viscous dissipation in flow through heated microchannels in order 
to establish a correlation between the Brinkman number and the Nusselt number. They found that viscous dissipation counteracts the gains of high heat transfer coefficients associated with a reduction in the channel size in a micro-channel design.

In many modern energy and biomedical systems, electro-kinetic flows arise. These constitute flows in which charged particles or ionic fluids (electrolytes) are mobilized by electrical fields in conduits which have charged walls. A special case of electro-kinetic flow is electro-osmotic flow where the electrostatic body force is large in the electrical double layers, but the proximity of the charged no-slip surface leads to significant viscous drag. Electro-osmotic flow is therefore induced by an applied potential or electric field in a system that has an imbalance of charge (e.g. charged walls). It is important to distinguish electrokinetic and electro-osmotic flows from electrohydrodynamic flows which usually apply to only weakly conducting dielectric liquids. Electro-osmotic flows arise both naturally (e.g. in blood flows) and can be induced artificially in a variety of energy and chemical process applications. They feature an electrical double layer adjacent to the conduit boundary. Regions featured are separated by a slip surface which is locally well-approximated by the Helmholtz-Smoulochowski relation. Common assumptions in modelling electro-osmotic flows are constant wall surface electrical charges, neglection of streaming currents, high zeta potentials (i.e. large values of electrical potential at the shear plane of the electric double layer) and fully-developed hydrodynamic flow. The Debye-Hückel linear approximation is also frequently employed in electro-osmotic flows (EOF) since it retains some of the physics (although it is valid only for lower electrical potentials) but greatly simplifies the mathematics. Without the Debye-Hückel linear approximation, the Poisson-Boltzmann equation generally requires a complete numerical solution. An electro-osmotic flow of non-Newtonian fluids (power-law model) through a curved rectangular microchannel was studied by Nekoubin [15]. He used central difference scheme to solve the PoissonBoltzmann equation without imposing the Debye-Hückel linear approximation. Medina et al. [16] investigated mathematically the species diffusion of a neutral solute in a pulsatile electro-osmotic flow (PEOF), circulating in a parallel flat plate microchannel with asymmetric zeta potentials imposed at the walls. Santiago [17] conducted an excellent study of electro-osmotic hydrodynamics in micro-channels, considering in detail the outer flow region, which, unlike the inner flow region (dominated by viscous and electro-static forces) is controlled by pressure, inertia, and non-uniform surface charge. 
Haywood et al. [18] reported both nonlinear numerical simulations and experimental results for electro-osmotic mobilities in nanofluidic channels with rectangular cross sections. Other interesting studies include Dutta et al. [19] (for microelectromechanical systems control), Kim et al. [20] (who also presented microscopic particle image velocimetry measurements) and Liang et al. [21] (the rotating electroosmotic flow (EOF) of Oldroyd-B fluid in a microchannel under high zeta potential).

An important pumping mechanism which features in many biological systems is peristalsis. Peristaltic micro-pumps have also been deployed to excellent effect in the precise dosing and dispensing of aggressive, hot, corrosive or even sterile fluid media. To achieve robust characterization of the hydrodynamics of peristaltic micro-pumps and biological flows, engineers have developed an extensive range of analytical and computational models $[22,23,24,25]$. In energy applications, both thermal convection and efficiency can be passively enhanced by modifying the flow geometry and boundary conditions, e.g. inlet and wall conditions or alternatively by enhancing working fluid thermophysical properties [26, 27, 28].

The above studies generally considered straight micro-channels. Curvature however, is common in many energy (and physiological) applications. Both theoretical and computational fluid dynamic aspects including channel aspect ratio and radius ratio for curved micro-channels have been described in detail by Al-Halhouli et al. [29]. Numerous applications of curved microchannel systems have been explored in the literature [30, 31, 32, 33]. Moreover, there have been a couple of investigations for entropy generation on electro-kinetically modulated peristaltic transport with the Newtonian and non-Newtonian fluids $[34,35,36]$.

In the present work, a theoretical study is described for electro-osmotic peristaltic pumping of ionic nanofluids in a curved deformable microchannel. The transformed conservation equations are simplified for the case of low zeta potential (Debye Hückel linearization). The linearized Poisson-Boltzmann equation is solved analytically. With the aid of Mathematica software, the influence of various emerging parameters on velocity, temperature, nanoparticle concentration profiles is computed. Micro-channel curvature effects on the stream line patterns, temperature contours and nanoparticles concentration contours are also computed. The study is relevant to hybrid electrokinetic nanofluid micro-channels in novel nanoscale energy applications. 


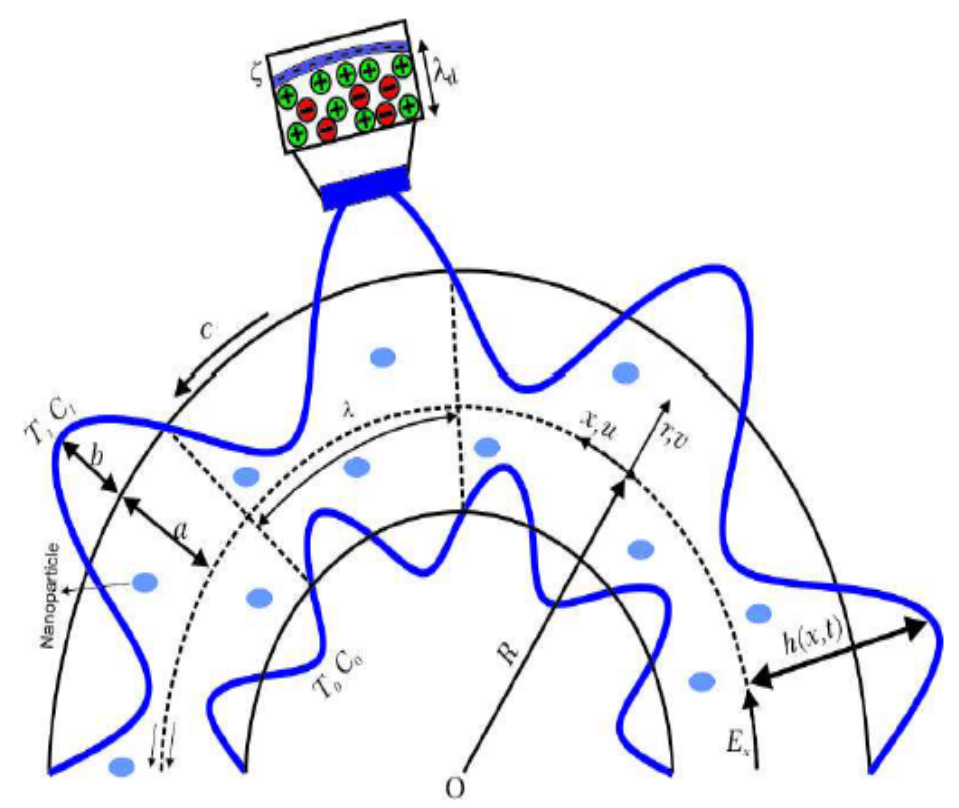

Figure 1: Physical model of peristaltic pumping of electro-osmotic nanofluid in a curved micro-channel

\section{Mathematical model for electro-osmotic nanofluid micro-pump}

We consider an incompressible nanofluid moving in a deformable curved micro channel with constant centerline radius $R$ and diameter $2 a$. We choose intrinsic coordinates $(x, r)$ in the laboratory frame with a velocity vector $\bar{V}=u(x, r, t) \widehat{e}_{x}+v(x, r, t) \widehat{e}_{r}$ as shown in Fig. 1. The flow is generated due to both the electrokinetic force and the transverse deflections of sinusoidal waves of small amplitude $b$ that are imposed on the flexible walls of the channel. The lower wall of the channel is maintained at constant temperature $T_{0}$, and concentration $C_{0}$. Similarly, the upper wall of the channel is maintained at constant temperature $T_{1}$, and concentration $C_{1}$. The equations of the deformable micro-channel walls are described as:

$$
r= \pm h(x, t)= \pm\left(a-b \cos ^{2} \pi\left(\frac{x-c t}{\lambda}\right)\right),
$$

Here, $x$ is the axial distance, $a$ the radius of the stationary curved channel, $\lambda$ the wave length, $t$ the time, $c$ the wave velocity, and $h$ the radial displacement of the wave from the centerline. The wavelength is large compared with the channel's width $\left(\frac{a}{\lambda}<<1\right)$. 


\subsection{Conservation equations for electro-osmotic nanofluid transport}

Viscous, incompressible, Newtonian, electro-osmosis modulated peristaltic transport of ionic nanofluids through a finite curved micro-channel is considered (Fig. 1). The appropriate governing equations for the conservation of mass, momentum, energy and nanoparticle (species) concentration for the regime can be written as:

equation of mass conservation

$$
\frac{R}{r+R} \frac{\partial u}{\partial x}+\frac{\partial v}{\partial r}+\frac{v}{r+R}=0,
$$

equations of momentum conservation

$$
\begin{aligned}
& \rho_{e f f}\left[\frac{\partial u}{\partial t}\right.\left.+(\bar{V} \cdot \nabla) u+\frac{u v}{r+R}\right]=-\frac{R}{r+R} \frac{\partial p}{\partial x}+\mu_{e f f}\left[\left(\frac{R}{r+R}\right)^{2} \frac{\partial^{2} u}{\partial x^{2}}\right. \\
&\left.+\frac{1}{r+R} \frac{\partial u}{\partial r}+\frac{\partial^{2} u}{\partial r^{2}}-\frac{u}{(r+R)^{2}}+\frac{2 R}{(r+R)^{2}} \frac{\partial v}{\partial x}\right] \\
&+\rho_{\text {eff }} g\left(\beta_{t}\left(T-T_{0}\right)+\beta_{c}\left(C-C_{0}\right)\right)+\rho_{e} E_{x}, \\
& \rho_{e f f}\left[\frac{\partial v}{\partial t}+(\bar{V} \cdot \nabla) v-\frac{u^{2}}{r+R}\right]=-\frac{\partial p}{\partial r}+\mu_{e f f}\left[\left(\frac{R}{r+R}\right)^{2} \frac{\partial^{2} v}{\partial x^{2}}\right. \\
&\left.+\frac{1}{r+R} \frac{\partial v}{\partial r}+\frac{\partial^{2} v}{\partial r^{2}}-\frac{v}{(r+R)^{2}}-\frac{2 R}{(r+R)^{2}} \frac{\partial u}{\partial x}\right]+\rho_{e} E_{r},
\end{aligned}
$$

equation of energy (heat) conservation

$$
\begin{aligned}
& \left(\rho c_{p}\right)_{\text {eff }}\left[\frac{\partial T}{\partial t}+(\bar{V} \cdot \nabla) T\right]=k_{e f f} \nabla^{2} T+\frac{D K_{T}}{C_{S}} \nabla^{2} C+\mu_{e f f}\left[2 \left\{\left(\frac{\partial v}{\partial r}\right)^{2}\right.\right. \\
& \left.\left.+\left(\frac{R}{r+R} \frac{\partial u}{\partial x}+\frac{v}{r+R}\right)^{2}\right\}+\left(\frac{\partial u}{\partial r}+\frac{R}{r+R} \frac{\partial v}{\partial x}-\frac{u}{r+R}\right)^{2}\right]+\sigma_{e f f} E_{x}^{2}
\end{aligned}
$$

Equation of nano-particle species conservation

$$
\left[\frac{\partial C}{\partial t}+(\bar{V} \cdot \nabla) C\right]=D \nabla^{2} C+\frac{D K_{T}}{T_{m}} \nabla^{2} T,
$$

where the vector operators $\bar{V} \cdot \nabla$ and $\nabla^{2}$ are given in the appendix Appendix A. The parameters $p, \rho_{e f f}, \beta_{t}, \beta_{c}, \mu_{e f f}, k_{e f f}, c_{p}, \sigma, E, T, C, D, K_{T}, C_{S}$, and $T_{m}$ are pressure, density, coefficient of thermal expansion, coefficient of expansion with concentration, dynamic viscosity, thermal conductivity, 
specific heat at constant pressure, electrical conductivity, applied electrical field, temperature, concentration, mass diffusivity, thermal diffusion ration, concentration susceptibility, and mean fluid temperature, here the subscript $(e f f)$ refers to the effective property of nanofluids. The electro-osmotic body force terms in the above momentum equations is given as

$$
\mathrm{E}=\rho_{e}\left(E_{x} \widehat{e}_{x}+E_{r} \widehat{e}_{r}\right)=-\rho_{e}\left(\frac{R}{(R+r)} \frac{\partial \Phi}{\partial x} \widehat{e}_{x}+\frac{\partial \Phi}{\partial r} \widehat{e}_{r}\right),
$$

where $\rho_{e}=e\left(z_{+} n_{+}-z_{-} n_{-}\right)$is the net charge density of the aqueous medium of permittivity $\varepsilon, e$ is the elementary electronic charge, here $z_{ \pm}$and $n_{ \pm}$are the valence and number densities of co-ions and counterions respectively, and $\Phi$ is the electric potential. The third term and the fourth term on the right-hand side of equation (5) represent respectively the local volumetric heating due to viscous dissipation and volumetric heat generation due to electric resistance heating (Joule heating). The important correlations of thermo-physical properties of nanofluids are given in the appendix Appendix B.

\subsection{Potential distribution inside the electrical double layer (EDL)}

According to electrostatics theory (see Melcher [37]), the electric potential, $\Phi$, is governed by well-known Poisson equation,

$$
\nabla^{2} \Phi=-\frac{\rho_{e}}{\varepsilon_{e f f}} .
$$

In general, the ion distribution is affected by two different contributions: $(a)$ the externally applied potential, $\tilde{\Phi}$, and $(b)$ the electrical double layer (EDL) potential, $\Psi$, associated surface zeta-potential, $\zeta$. However, it is important to mention that, while the externally applied potential is presented by Laplace equation, the equation (8) with net charge density in a unit volume of a symmetric electrolyte, i.e. $n_{+0}=-n_{-0}=n_{0}, z_{+}=z_{-}=z$, can be decomposed as

$$
\nabla^{2} \tilde{\Phi}=0, \quad \text { and } \quad \nabla^{2} \Psi=-\frac{z e}{\varepsilon_{e f f}}\left(n_{+}-n_{-}\right) .
$$

where $n_{+}$and $n_{-}$are positive and negative ions having bulk concentration (number density) $n_{0}$ and $z$ is the valence of the ions. Here, The electrical permittivity of composite medium (electrolytic nanofluid), $\varepsilon_{\text {eff }}$ is defined by the following relation (Chakraborty and Roy [38]):

$$
\varepsilon_{e f f}=\varepsilon_{f}+3 \alpha \varepsilon_{f}\left(\frac{\varepsilon_{s}-\varepsilon_{f}}{\varepsilon_{s}+2 \varepsilon_{f}-\alpha\left(\varepsilon_{s}-\varepsilon_{f}\right)}\right) .
$$


To define potential distribution, it is important to analyze the nano-particle species conservation equation. The Nernst-Planck equation for the curved microchannel geometry in the absence of chemical reactions can be expressed as

$$
\begin{aligned}
& \left(\frac{\partial n_{ \pm}}{\partial t}+u \frac{R}{R+r} \frac{\partial n_{ \pm}}{\partial x}+v \frac{\partial n_{ \pm}}{\partial r}\right)=D_{ \pm}\left(\nabla^{2} n_{ \pm}\right) \\
& \quad \pm \frac{D_{ \pm} z_{ \pm} e}{k_{B} T}\left[\frac{R}{R+r} \frac{\partial}{\partial x}\left\{n_{ \pm} \frac{R}{R+r} \frac{\partial \Psi}{\partial x}\right\}+\frac{1}{R+r} \frac{\partial}{\partial r}\left\{(R+r) n_{ \pm} \frac{\partial \Psi}{\partial r}\right\}\right]
\end{aligned}
$$

Intrinsic to the analysis is the assumption that there are equal ionic diffusion coefficients for both the species, and that the mobility of the species is given by the Einstein formula and $D$ is the diffusivity of an ionic species.

\subsection{Non-dimensional scaling}

The governing conservation equations can be greatly simplified by applying lubrication hydrodynamic theory approximations. It is therefore assumed that Reynolds number $(R e)$ and the local slope of the micro-channel wall are very small, which yields a micro-channel with negligible inertial effects $(\lambda / a \gg 1)$. To facilitate analytical solutions, the following dimensionless variables and parameters are invoked:

$$
\begin{aligned}
& x^{\prime}=\frac{x}{\lambda}, r^{\prime}=\frac{r}{a}, u^{\prime}=\frac{u}{c}, v^{\prime}=\frac{v}{\delta c}, \delta=\frac{a}{\lambda}, t^{\prime}=\frac{c t}{\lambda} h^{\prime}=\frac{h}{a}, \phi=\frac{b}{a}, \kappa=\frac{R}{a}, \\
& L^{\prime}=\frac{L}{\lambda}, p^{\prime}=\frac{a^{2} p}{\mu c \lambda}, R e=\frac{\rho_{f} c a}{\mu}, \psi^{\prime}=\frac{\psi}{a c}, \Psi^{\prime}=\frac{\Psi}{\Psi_{0}}, \zeta^{\prime}=\frac{\zeta}{\Psi_{0}}, n^{\prime}=\frac{n}{n_{0}},
\end{aligned}
$$

in which $R e$ is the Reynolds number, $\delta$ is the wave number, $\phi$ is the amplitude ratio or the occlusion parameter, $\kappa$ is the curvature parameter, $\Psi_{0}=k_{B} T / e z$ is the thermal potential and the primed quantity is the dimensionless version of the non-primed quantity. The non-dimensional form of the Nernst-Planck equation (11) is written as (after omitting all the primes)

$$
\begin{gathered}
P e \delta^{2}\left[u \frac{\kappa}{\kappa+r} \frac{\partial n_{ \pm}}{\partial x}+v \frac{\partial n_{ \pm}}{\partial r}\right]=\left(\delta^{2}\left(\frac{\kappa}{r+\kappa}\right)^{2} \frac{\partial^{2} n_{ \pm}}{\partial x^{2}}+\frac{\partial^{2} n_{ \pm}}{\partial r^{2}}+\frac{1}{r+\kappa} \frac{\partial n_{ \pm}}{\partial r}\right) \\
\pm\left(\delta^{2} \frac{\kappa}{\kappa+r} \frac{\partial}{\partial x}\left\{n_{ \pm} \frac{\kappa}{\kappa+r} \frac{\partial \Psi}{\partial x}\right\}+\frac{1}{\kappa+r} \frac{\partial}{\partial r}\left\{(\kappa+r) n_{ \pm} \frac{\partial \Psi}{\partial r}\right\}\right),
\end{gathered}
$$

here, $P e=\frac{c \lambda}{D}$ is the Péclet number which magnitude may range from $\sim$ $0.01-1$ for the typical values assumed as $c \sim 10^{-2} \mathrm{~m} / \mathrm{s}, \lambda \sim 10 \mu \mathrm{m}$, and 
$D \sim 10^{-9} \mathrm{~m}^{2} / \mathrm{s}$. The non-linear terms in the equation (12) are of $O\left(P e \delta^{2}\right)$. Therefore, for $\delta \rightarrow 0$ and $P e \delta^{2} \rightarrow 0$, the reduced form of the Nernst-Planck equation can be written as

$$
\frac{\partial^{2} n_{ \pm}}{\partial r^{2}}+\frac{1}{r+\kappa} \frac{\partial n_{ \pm}}{\partial r} \pm \frac{1}{\kappa+r} \frac{\partial}{\partial r}\left\{(\kappa+r) n_{ \pm} \frac{\partial \Psi}{\partial r}\right\}=0,
$$

subject to bulk conditions $n_{ \pm}(\Psi=0)=1$ and $\frac{\partial n_{ \pm}}{\partial r}=0$ where $\frac{\partial \Psi}{\partial r}=0$. The solution of equation (13) gives the Boltzmann distributions for the ions as

$$
n_{ \pm}=\exp (\mp \Psi) .
$$

Combining equations (9) and (14), we obtain the well-known non-linear Poisson-Boltzmann equation (in non-dimensional form), which yields the electric potential distribution in the EDL, i.e,

$$
\delta^{2}\left(\frac{\kappa}{r+\kappa}\right)^{2} \frac{\partial^{2} \Psi}{\partial x^{2}}+\frac{1}{r+\kappa} \frac{\partial}{\partial r}\left\{(r+\kappa) \frac{\partial \Psi}{\partial r}\right\}=m^{2} \sinh (\Psi),
$$

Where, $m$ is Debye-Hückel parameter, associated with thickness of the Debye layer (EDL thickness) $\lambda_{d}$ as $m=\frac{a}{\lambda_{d}}=\sqrt{\frac{2 n_{0} a^{2} e^{2} z^{2}}{\varepsilon_{e f f} k_{B} T}}$.

Proceeding to the classical lubrication theory framework $\left(\delta^{2} R e \rightarrow 0\right.$, $\delta \rightarrow 0$ ), the momentum equations (3) and (4) after eliminating the pressure term, the energy equation (5), the nanoparticles concentration equation (6), and Poisson-Boltzmann equation (15) in terms of the above dimensionless parameters and stream function $u=\frac{\partial \psi}{\partial r}, v=-\frac{\kappa}{r+\kappa} \frac{\partial \psi}{\partial x}$, after omitting the primes, emerge as:

$$
\begin{gathered}
\frac{\partial}{\partial r}\left[\frac{1}{r+\kappa} \frac{\partial}{\partial r}\left\{(r+\kappa)^{2}\left(\frac{\partial^{2} \psi}{\partial r^{2}}-\frac{1}{r+\kappa} \frac{\partial \psi}{\partial r}\right)\right\}+(r+\kappa)(G r \theta+G c \sigma)\right] \\
=-U_{H S} \frac{\partial^{2}}{\partial r^{2}}\left\{(r+\kappa) \frac{\partial \Psi}{\partial r}\right\} \\
\left(\frac{\partial^{2} \theta}{\partial r^{2}}+\frac{1}{r+\kappa} \frac{\partial \theta}{\partial r}\right)+B r\left(\frac{\partial^{2} \psi}{\partial r^{2}}-\frac{1}{r+\kappa} \frac{\partial \psi}{\partial r}\right)^{2}+\frac{P r D u}{r+\kappa} \frac{\partial}{\partial r}\left\{(r+\kappa) \frac{\partial \sigma}{\partial r}\right\}+\gamma=0 \\
\frac{\partial^{2} \sigma}{\partial r^{2}}+\frac{1}{r+\kappa} \frac{\partial \sigma}{\partial r}+\frac{S c S r}{r+\kappa} \frac{\partial}{\partial r}\left\{(r+\kappa) \frac{\partial \theta}{\partial r}\right\}=0 \\
\frac{\partial^{2} \Psi}{\partial r^{2}}+\frac{1}{r+\kappa} \frac{\partial \Psi}{\partial r}-m^{2} \sinh (\Psi)=0 .
\end{gathered}
$$


Here the new non-dimensional parameters introduced are $\theta=\frac{T-T_{0}}{T_{1}-T_{0}}, \sigma=$ $\frac{C-C_{0}}{C_{1}-C_{0}}, G r=\frac{\beta_{t g} \rho_{e f f}^{2} a^{3}\left(T_{1}-T_{0}\right)}{\mu_{e f f}}, G c=\frac{\beta_{c g \rho_{e f f}^{2} a^{3}\left(C_{1}-C_{0}\right)}}{\mu_{e f f}}, D u=\frac{D K_{T}\left(C_{1}-C_{0}\right)}{\mu_{e f f} c_{P} C_{S}\left(T_{1}-T_{0}\right)}$, $S r=\frac{\rho_{e f f} D K_{T}\left(T_{1}-T_{0}\right)}{\mu_{e f f} T_{m}\left(C_{1}-C_{0}\right)}, S c=\frac{\mu_{e f f}}{\rho_{e f f} D}, \operatorname{Pr}=\frac{\mu_{e f f} c_{p}}{k_{e f f}}, B r=\frac{\mu_{e f f} c^{2}}{k_{e f f}\left(T_{1}-T_{0}\right)}, \gamma=\frac{\sigma_{\text {eff }} E_{x}^{2} a^{2}}{k_{\text {eff }}\left(T_{1}-T_{0}\right)}$, and $U_{H S}=-\frac{\epsilon_{e} \Psi_{0} E_{x}}{c \mu_{e f f}}$. These denote respectively dimensionless temperature, dimensionless solute concentration, thermal Grashof number, nano-particle solutal Grashof numbers, Dufour number, Soret number, Schmidh number, Prandtl number, Brinkman number, the normalized generation term (ratio of Joule heating to surface heat flux, for constant wall temperature), and Helmholtz-Smoluchowski velocity (i.e. maximum electro-osmotic velocity). The associated imposed dimensionless boundary conditions at the walls of the curved micro-channel are given by:

$$
\begin{aligned}
& \psi=-\frac{F}{2}, \frac{\partial \psi}{\partial r}=0, \theta=0, \sigma=0, \Psi=\zeta_{1} \text { at } y=-h \\
& \psi=\frac{F}{2}, \frac{\partial \psi}{\partial r}=0, \theta=1, \sigma=1, \Psi=\zeta_{2} \text { at } y=h
\end{aligned}
$$

The non-dimensional wall deformation equation takes the form $h=1-$ $\phi \cos ^{2} \pi(x-t)$.

\section{Analytical solution for low zeta potentials}

The electric potential and ion distributions are independent of fluid velocity. For small zeta potentials $\left(\zeta_{i} \leq 25 \mathrm{mV}\right)$, the equation (19) can be linearized via a Taylor series expansion. This leads in the Debye-Hückel approximation and the simplified dimensionless Poisson-Boltzmann equation with corresponding boundary conditions take the form:

$$
\frac{1}{r+\kappa} \frac{\partial}{\partial r}\left[(r+\kappa) \frac{\partial \Psi}{\partial r}\right]=m^{2} \Psi, \quad \Psi(-h)=\zeta_{1}, \Psi(h)=\zeta_{2} .
$$

The solution of the boundary value problem in Eq. (21) can be obtained as

$$
\Psi(x, r)=\frac{\zeta_{1}\left[\left(K_{2}-R_{\zeta} K_{1}\right) I_{0}[m(r+\kappa)]+\left(R_{\zeta} I_{1}-I_{2}\right) K_{0}[m(r+\kappa)]\right.}{I_{1} K_{2}-I_{2} K_{1}} .
$$

Here, $I_{0}$ and $K_{0}$ are modified Bessel functions of first and second kind of order 0 respectively, $I_{1}=I_{0}[m(k-h)], I_{2}=I_{0}[m(k+h)], K_{1}=K_{0}[m(k-h)]$ and $K_{2}=K_{0}[m(k+h)]$. 


\section{Computational results and discussion}

Numerical evaluation of the closed-form solutions for velocity, temperature, nano-particle concentration (as described earlier), is conducted in Mathematica 9 NDSolve algorithm. Streamline, temperature and nano-particle concentration contours are also computed. The results are visualized graphically for the influence of selected parameters in Figs. 2-8. For brevity, we restrict the discussion to the influence of six selected parameters, namely Brinkman Number $(B r)$, Joule heating parameter $(\gamma)$, maximum electroosmotic velocity $\left(U_{H S}\right)$, inverse EDL thickness parameter $(m)$, micro-channel curvature parameter $(\kappa)$ and zeta potential ratio $\left(R_{\zeta}\right)$. The maximum electroosmotic velocity, i.e. Helmholtz-Smoluchowski velocity $\left(U_{H S}\right)$ is a key parameter examined here. This characterizes the effect of applied external electric field (if there is no electric field $U_{H S}=0$ and the model reduces to the peristaltic flow of non-conducting nanofluid through a curved microchannel) on velocity profile. We further note that throughout, a constant axial pressure gradient is studied and a finite thickness of the Debye electrical double layer effects is examined. The Joule heating parameter $(\gamma)$ is proportional to the effect of the nanoparticle volume fraction and also proportional to the diameter of nanoparticles. Nano-particle geometry effects are not explicitly considered.

\subsection{Hydrodynamic analysis}

Figures 2(a)-2(d) illustrate the evolution in velocity versus radial coordinate in the micro-channel with a change in $(a)$ curvature parameter $\kappa,(b)$ maximum electro-osmotic velocity $U_{H S},(c)$ inverse EDL thickness parameter $m,(d)$ zeta potential ratios $R_{\zeta}$. The case of a straight micro-channel is retrieved for $\kappa \rightarrow \infty$. A progressive decrease arises in velocity with increasing curvature parameter (Fig. 2(a)) as we progress from one microchannel wall (at $r=-0.6$ ) to the centerline of the microchannel. However, with the progress from the centerline to the opposite wall, i.e. over the range $0<r<0.6$ the opposite effect is induced. Evidently there is an intimate connection between the location across the cross-section and the influence of curvature parameter. Closer to the inner wall $(r=-0.6)$ curvature effects become dominant and acceleration is induced in the flow. Peak velocity arises near the centerline. Curvature effect is therefore non-trivial and modifies the velocity distribution. It is clear from these figures that different responses for each parameter are induced in the two sections of the 
microchannel i.e. the lower half space $(-0.6<r<0)$ and the upper half space $(0<r<0.6)$. Since $G r=5, G c=1$, the thermal buoyancy effect has been five times that of the species (nano-particle concentration) buoyancy effect. Furthermore $D u=2, S r=1$, implies that the Dufour diffusion is twice that of the Soret diffusion. These effects have been considered already in existing studies of peristaltic nanofluid propulsion and are therefore not repeated here. All computations are considered at a specific axial location, i.e. $x=0.2$ and at the inception of the flow, $t=0$. Fig. 2(b) shows that a decrease in maximum electro-osmotic velocity $U_{H S}$, generates a deceleration in the flow in the lower microchannel half space whereas the opposite effect is induced in the upper half space. In the latter maximum velocity corresponds to the highest value of $U_{H S}=+1$ whereas in the former zone the minimum velocity computed is associated with $U_{H S}=+1$. The peak velocity computed anywhere is displaced, however further from the centerline and is closer to the upper microchannel wall $(r=0.6)$. Fig. 2(c) shows that with an increase in inverse EDL thickness parameter $(m)$ i.e. electro-osmotic parameter, there is a significant enhancement in nanofluid velocity in the lower microchannel half space whereas the reverse trend is generated in the upper microchannel half space. The electro-osmotic parameter is inversely proportional to the Debye length or characteristic thickness of the electrical double layer (EDL). Reducing Debye length, i.e. increasing $m$ value, therefore manifests in increasing the electrical potential, as noted in Datta and Choudhary [39]. The electrical potential diminishes with every increase in Debye length since a greater quantity of ions takes the place of the counter ions as we progress away from the charged surface. Debye length is therefore a critical design parameter in controlling the electrical potential distribution. As simulated via the inverse EDL thickness parameter, it is found that velocity can be considerably manipulated in both regions of the microchannel. The velocity profiles with variation in $m$ are also found to be more evenly distributed in both sections of the micro-channel i.e. they are not as sharp as in Figs. 2(a) and 2(b). In Fig. 2(d) an elevation in the zeta potential ratio $R_{\zeta}\left(=\zeta_{2} / \zeta_{1}\right)$ is examined. This parameter embodies the ratio of the zeta potentials imposed on the upper and lower microchannel walls. With an increase in positive zeta potential ratio the flow is significantly decelerated in the lower microchannel half space, whereas with an increase in negative zeta potential significant flow acceleration is induced in the same zone. The converse response is obtained in the upper channel half space. The implication is that with higher zeta potential ratio the regime is stabilized, and this 


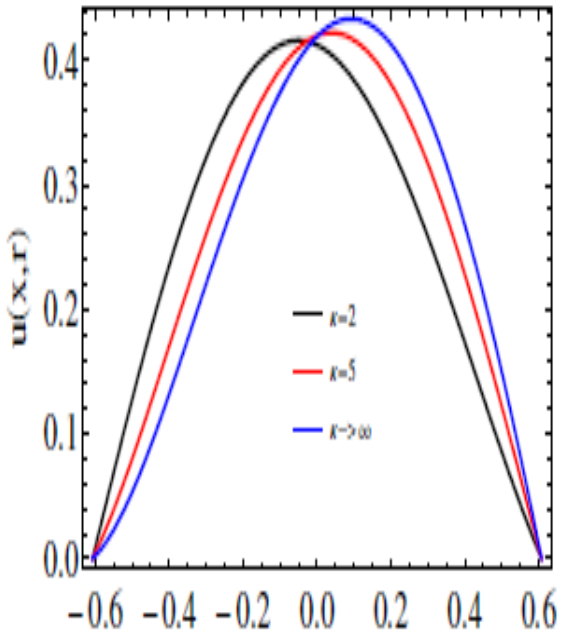

(a)

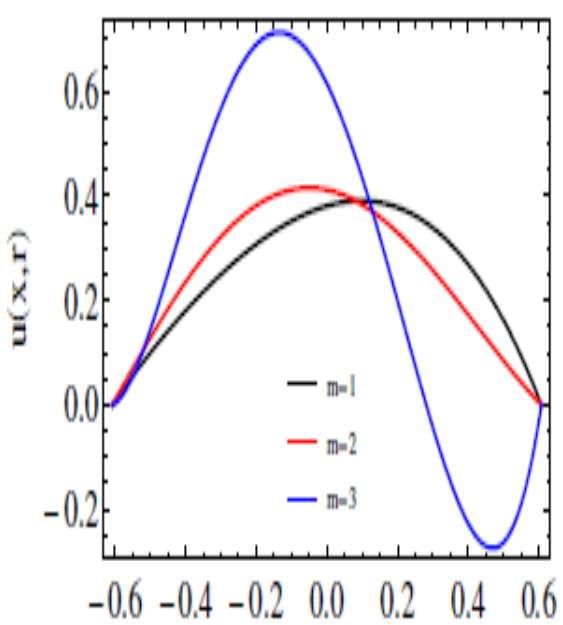

(c)

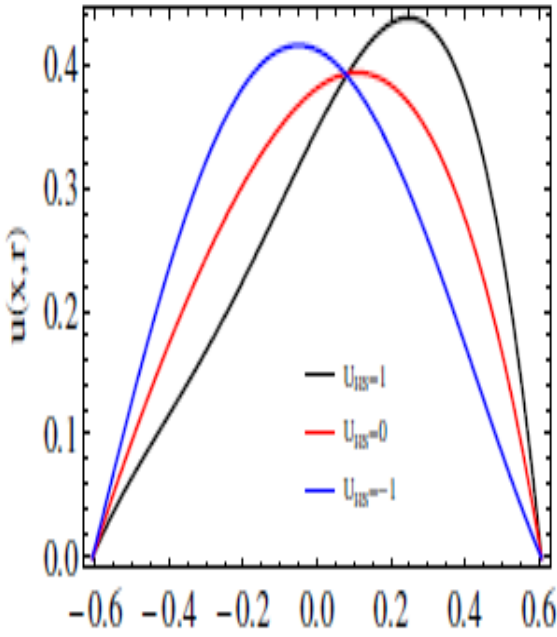

(b)

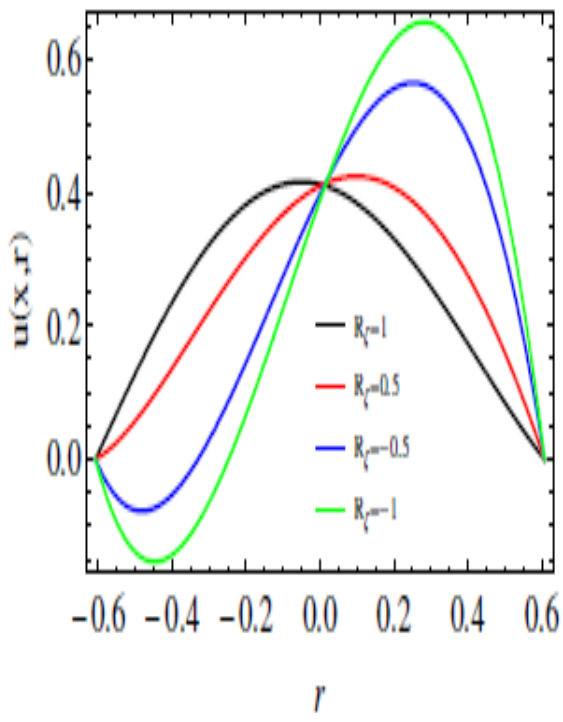

(d)

Figure 2: Velocity profiles for variation in different parameters (a) curvature parameter k, (b) electro-osmotic velocity $U_{H S}$, (c) inverse EDL thickness parameters $m$, (d) zeta

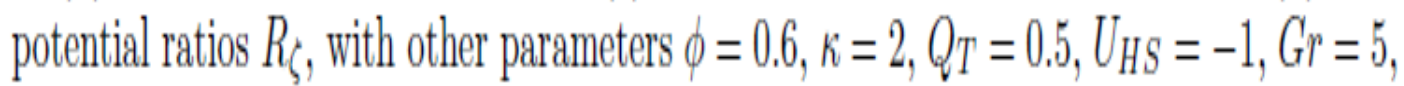
$G_{c}=1, D u=2, S_{r}=1, S_{c}=1, m=2, \gamma=2, R_{\zeta}=1, x=0.2, t=0$. 
is a characteristic effect associated with electro-osmotic flows, as noted by Kirby [40]. Nanofluids are colloids with nano-particles suspended in the ionic base fluid. Colloids with high zeta potential (negative or positive) are therefore electrically-stabilized. With a lower zeta potential ratio, coagulation or flocculation may arise which is undesirable in micro-channel applications in energy systems. The results show thatzeta potential ratio is a good indicator of the characterization of double-layer properties. Numerous studies have confirmed that the zeta potential quantifies the extent of electrostatic repulsion between adjacent, similarly charged particles in a nanofluid suspension or indeed in any other ionic colloid. For molecules and particles that are small enough, a high zeta potential will confer stability, i.e., the solution or dispersion will resist aggregation. When the potential is small, attractive forces may exceed this repulsion and the dispersion may break and flocculate. Russel et al. [41] have provided significant details on this aspect and the reader refers to their work for further elaboration. Fluid trapping can be analyzed

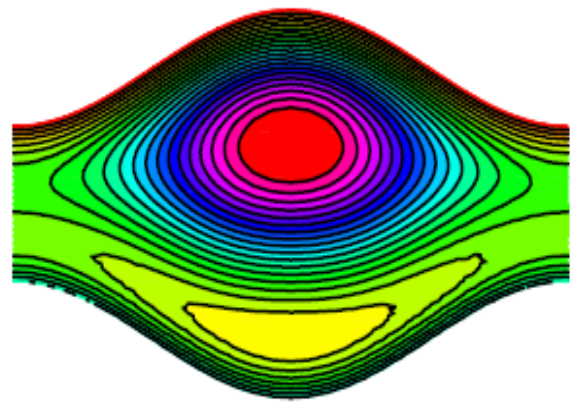

(a) $\kappa=1.5$

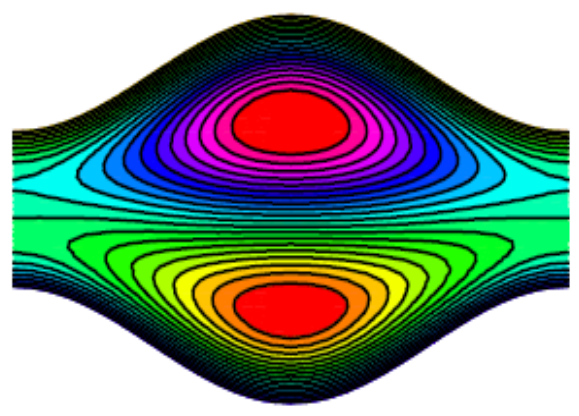

(c) $\kappa=5$

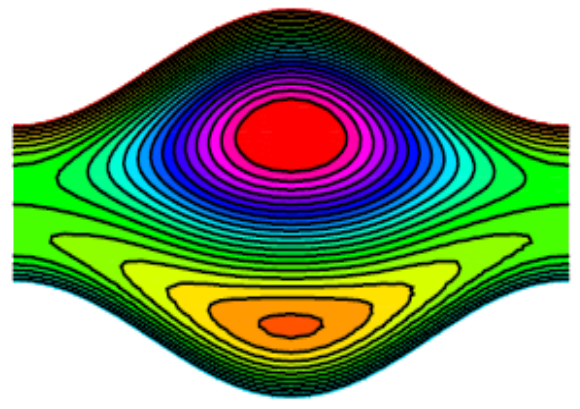

(b) $\kappa=2$

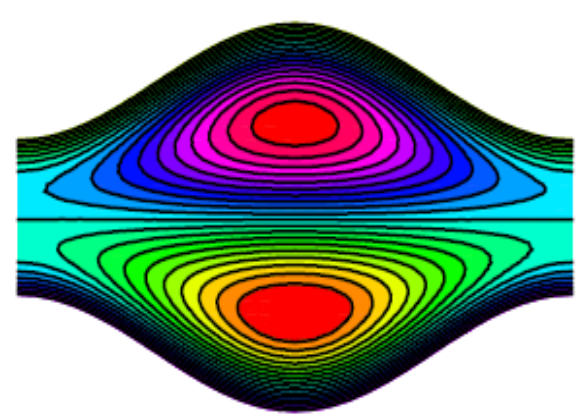

(d) $\kappa \rightarrow \infty$

Figure 3: Stream lines for channel curvature in wave frame of reference. The other parameters are $\phi=0.6, Q_{T}=1.2, B r=2, U_{H S}=-1, G r=1, G c=1, D u=0.5, S r=0.5$, $S c=1, m=2, \gamma=2, R_{\zeta}=-1, x=0.2, t=0$.

by plotting streamlines in a frame of reference that moves with the wave ve- 
locity. Fig. 3 shows a non-axial trapping of nanofluid in a micro channel with large curvature $(\kappa=2)$ and with small curvature $(\kappa \rightarrow \infty)$. In the non-axial fluid trapping there are two stagnation points with closed streamlines near the channel walls. The trapped particles of fluid comprise two asymmetrical boluses in a curved channel. The upper microchannel half space features a larger and more elliptical bolus whereas the lower micro-channel exhibits a distinctly smaller and more compressed bolus formation (see Figs. 3(a), $3(\mathrm{~b})$, and 3(c)). The trapped boluses are near symmetric when the channel curvature is small (i.e. straight channel). In other words, With decreasing channel curvature, the upper bolus is found to contract and the lower bolus is observed to expand (see Fig. 3(d)). Large curvature parameter (implying a tendency to a straighter geometry) encourages the establishment of similar bolus structures in the channel two half spaces. The presence of strong curvature (lower curvature value) indicates a lack of symmetry in the flow structure and this results in the discrepancy in bolus magnitude.

\subsection{Heat and mass transfer analysis}

Figures 4(a)-4(d) present temperature distributions versus radial coordinate in the micro-channel with a change in (a) Brinkman Number $\mathrm{Br}$ (b) Joule heating parameter $\gamma$ (c) maximum electro-osmotic velocity $U_{H S}$, (d) inverse EDL thickness parameter $m$. Unlike the plots in Figs 2(a)-2(d), the thermal and species buoyancy effects are constrained to be the same $(G r=1$ and $G c=1$ ) and furthermore, both Dufour and Soret cross-diffusion effects are equal $(D u=0.5$ and $S r=0.5)$. Zeta potentials at the upper and lower micro-channel walls are also identical $\left(R_{\zeta}=1\right)$. Furthermore a curved geometry is considered $(\kappa=2)$. In consistency with the imposed temperature boundary conditions, zero temperature is enforced at the lower microchannel wall and non-zero temperature at the upper micro-channel wall. Inspection of Fig. 4(a) shows that with the increasing Brinkman number there is a substantial elevation in temperature across the micro-channel span. Temperature values also generally ascend smoothly from the lower micro-channel wall and peak above the centerline in the upper channel zone. The Brinkman number, $B r=\frac{\mu_{e f f} c^{2}}{k_{e f f}\left(T_{1}-T_{0}\right)}$, embodies the ratio of heat produced by viscous dissipation and heat transported by molecular conduction. Larger values of $\mathrm{Br}$ imply a slower rate of conduction heat transfer generated by viscous dissipation and hence a greater temperature rise. Kinetic energy destroyed in the ionic nanofluid via viscous heating is dissipated as thermal energy which heats the nanofluid. The case of $\mathrm{Br}=0$ corresponds to neglect of viscous 


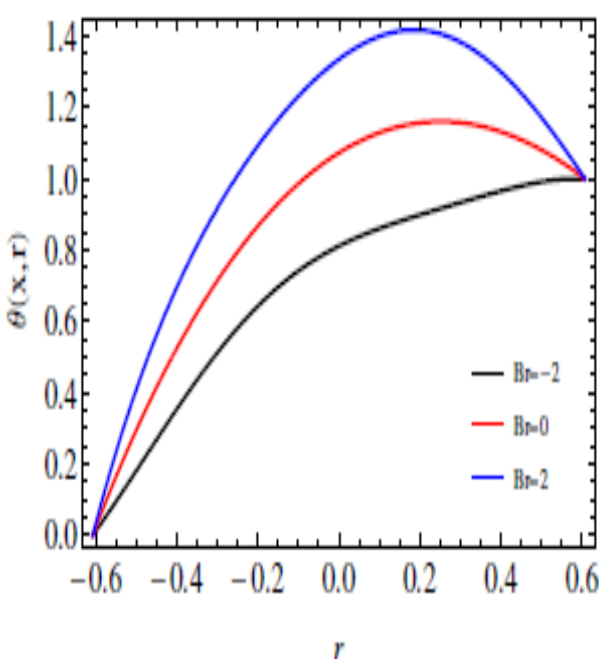

(a)

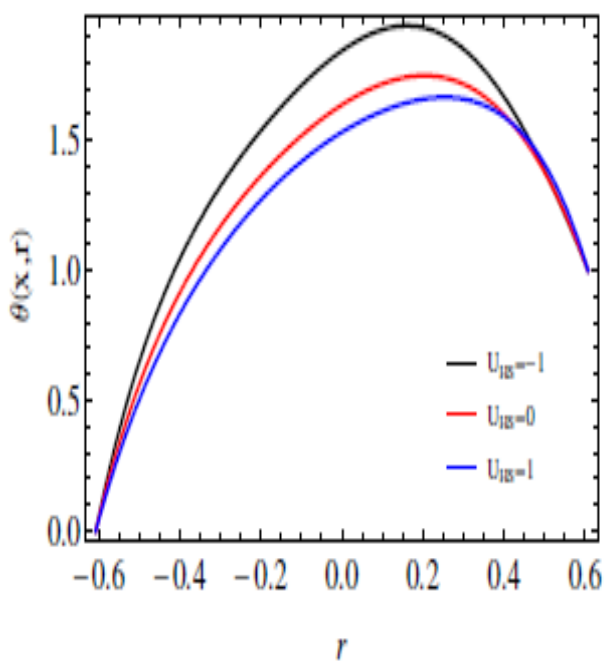

(c)

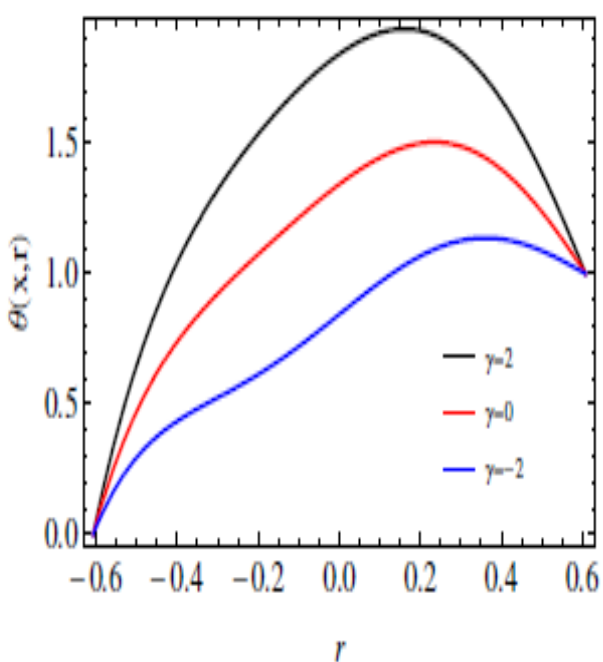

(b)

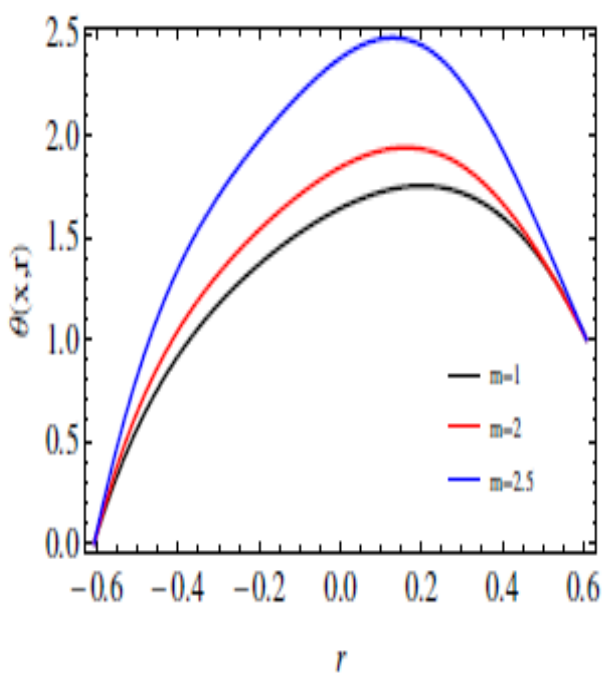

(d)

Figure 4: Temperature profiles for variation in different parameters (a) Brinkman Number $\mathrm{Br}$ (b) joule heating parameter $S(\mathrm{c})$ electro-osmotic velocity $U_{H S}$, (d) inverse EDL thickness parameters $m$, with other parameters $\phi=0.6, \kappa=2, Q_{T}=0.8, B r=2, U_{H S}=-1$, $G_{r}=1, G_{c}=1, D u=0.5, S r=0.5, S c=1, m=2, \gamma=2, R_{\zeta}=1, x=0.2, t=0$. 
heating effects and clearly significantly under-predicts the actual temperature in the electro-osmotic nanofluid flow. The computations agree with other investigations of ionic electro-osmotic flows, for example Ting et al. [42]. In Fig. 4(b), we depict the variation in the temperature as a function of the Joule heating parameter $(\gamma)$ for the three cases of $\gamma$, each depicting the case of absent, present and negative or present and positive. A negative value of $\gamma$ implies a notable reduction in the magnitude of the temperature across the microchannel whereas positive Joule heating parameter significantly raises the temperature. In other words, a negative value of Joule heating parameter results cooling and a positive value of $\gamma$ lead to heating in the electro-osmotic fluid flow through a microchannel. Furthermore, it is quite obvious in the electrokinetic flow through a microchannel, when the electric field is increased, the heat generation in the fluid also enhanced. Evidently the Joule electro-thermal parameter exerts a substantial influence on temperature evolution and the absence of this parameter in mathematical models may lead to either an under-prediction or over-prediction in actual temperatures. Fig. 4(c) shows that an increase in maximum electro-osmotic velocity $\left(U_{H S}\right)$ generates a reduction in temperature, whereas a decrease induces a significant heating in the ionic nanofluid. This trend is sustained across the micro-channel span. Fig. 4(d) indicates that with an elevation in inverse EDL thickness parameter $(m)$ i.e. electro-osmotic parameter, there is a notable boost in ionic nanofluid temperatures. Heating is therefore encouraged with lower electrical double layer thickness, whereas cooling is induced with higher electrical double layer thickness (lower inverse EDL thickness parameter value) in the regime.

Figures 5(a)-5(d) present nano-particle concentration profiles for $(a)$ Brinkman number $\mathrm{Br}(b)$ Joule heating parameter $\gamma(c)$ maximum electro-osmotic velocity $U_{H S},(d)$ inverse EDL thickness parameter $m$, again with equal thermal and species buoyancy forces $(G r=G c=1)$ and equal Dufour and Soret diffusion effects $(D u=0.5$ and $S r=0.5)$. Again, a curved microchannel scenario is considered $(\kappa=2)$. Very different distributions are computed as compared with the temperature profiles in Figs 4(a)-4(d). In all cases monotonic ascents from the lower microchannel wall to the upper microchannel wall (zero nano-particle concentration is enforced at the former with a maximum value imposed at the latter) are considered. An increase in Brinkman number (Fig. 5(a)) is observed to markedly depress nano-particle concentration magnitudes in the ionic nanofluid. This is the opposite effect to that 


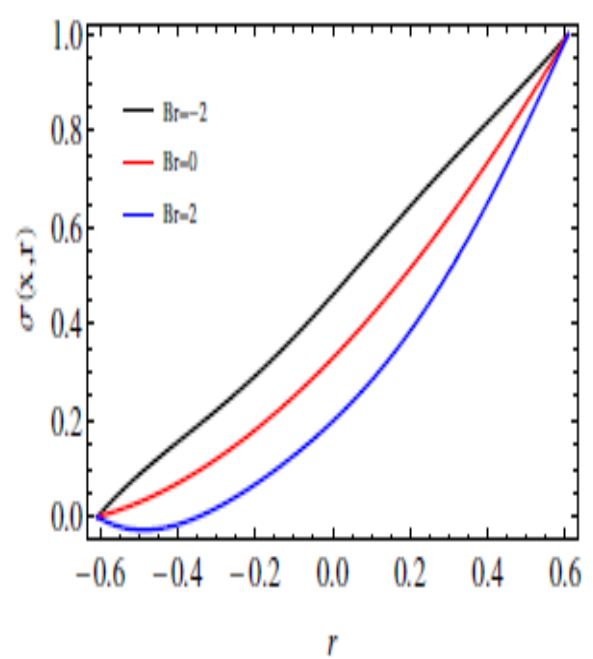

(a)

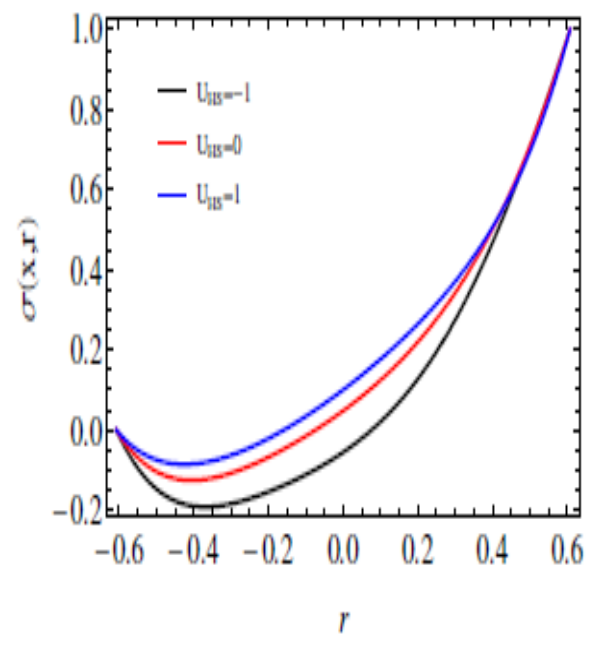

(c)

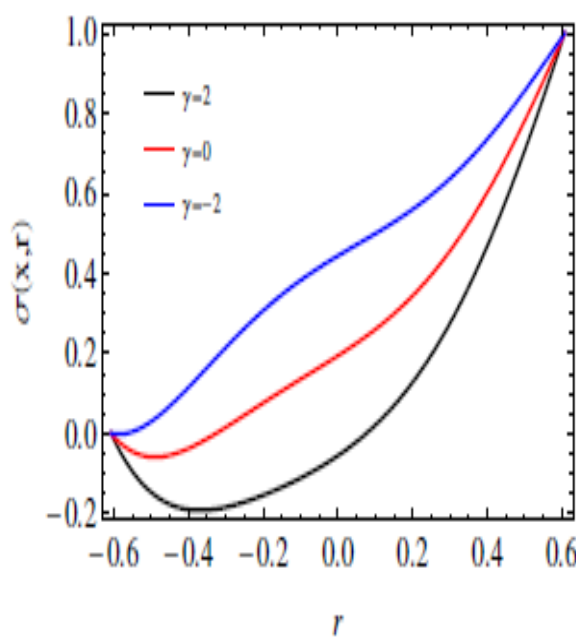

(b)

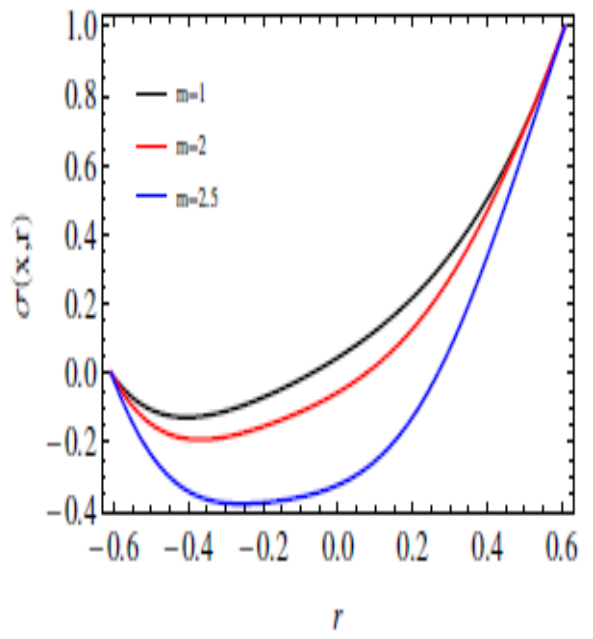

(d)

Figure 5: Concentration profiles for variation in different parameters (a) Brinkman Number $\mathrm{Br}$ (b) joule heating parameter $S$ (c) electro-osmotic velocity $U_{H S}$, (d) inverse EDL thickness parameters $m$, with other parameters $\phi=0.8, k=2, Q_{T}=0.5, B r=2$, $U_{H S}=-1, G r=1, G c=1, D u=0.5, S r=0.5, S c=1, m=2, \gamma=2, R_{\zeta}=1, x=0.2$, $t=0$. 
induced in the temperature field. The enhancement in temperatures serves to elevate thermal diffusion in the microchannel but counteracts the nanoparticle species diffusion resulting in lower concentration values across the microchannel span. Fig. 5(b) shows that with negative Joule heating parameter $(\gamma=-2)$ the nano-particle concentrations are boosted whereas they are suppressed with positive Joule heating parameter $(\gamma=2)$. Again, this is the opposite trend to that computed for the temperature distributions (Fig. 4b). In Fig. 5(c), increasing maximum electro-osmotic velocity (positive $U_{H S}$ ) is found to generate a weak increase in nano-particle concentration, whereas decreasing maximum electro-osmotic velocity (negative $U_{H S}$ ) induces a strong decrease in nano-particle concentrations. Fig. 5(d) shows that a marked reduction in nano-particle concentrations in the ionic nanofluid (across the microchannel span) accompanies an increase in electro-osmotic parameter $(m)$ i.e. a decrease in electrical double layer thickness. Figures 6-7 illustrate the

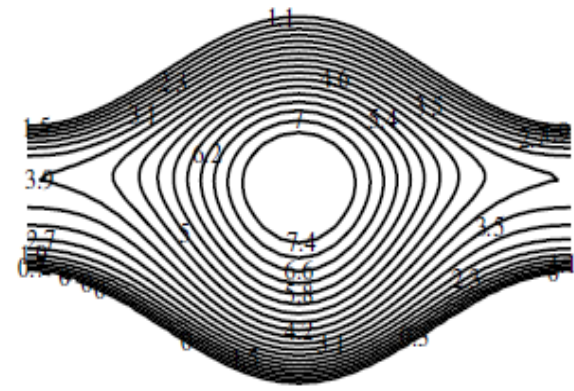

(a) $\kappa=1.5$

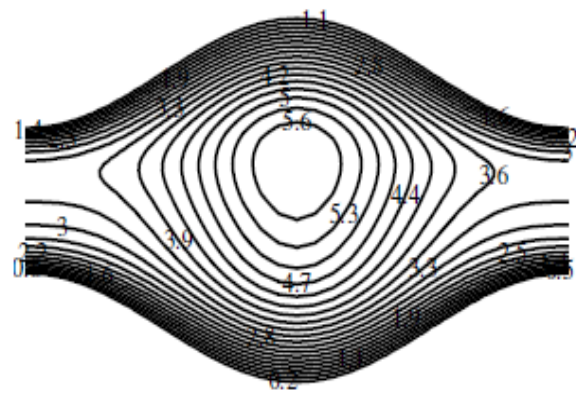

(c) $\kappa=5$

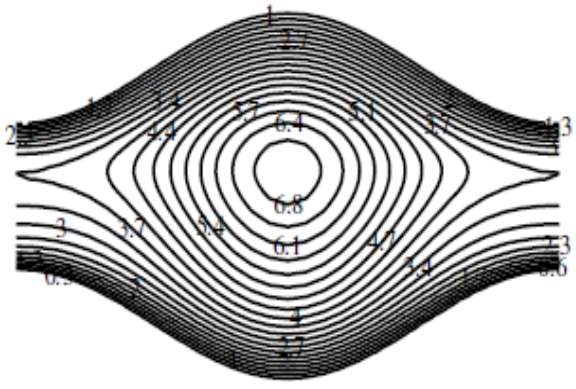

(b) $\kappa=2$

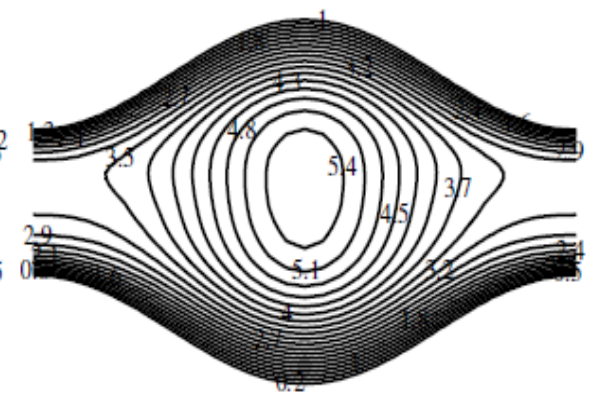

(d) $\kappa \rightarrow \infty$

Figure 6: Temperature contours for channel curvature. The other parameters are $\phi=0.6$, $Q_{T}=1.5, B r=2, U_{H S}=-1, G r=1, G c=1, D u=2, S r=1, S c=1, m=2, \gamma=2$, $m=2, R_{\zeta}=1$.

temperature and nano-particle concentration contours in the peristaltic microchannel flow with variation in the conduit curvature parameter $(\kappa)$. Figs 


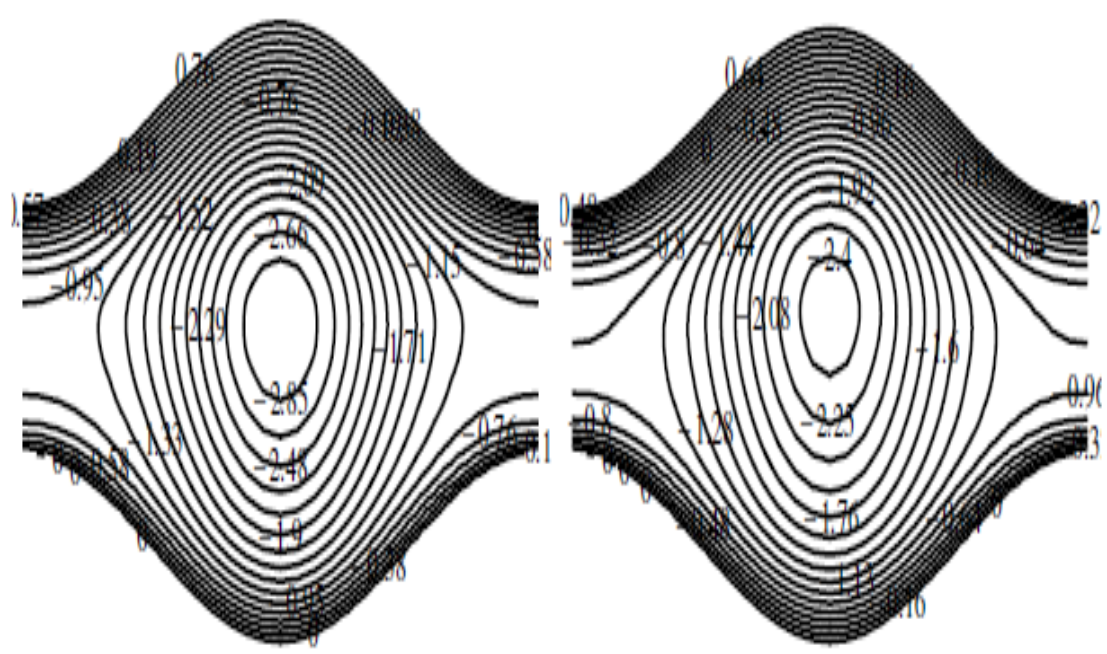

(a) $k=1.5$

(b) $k=2$

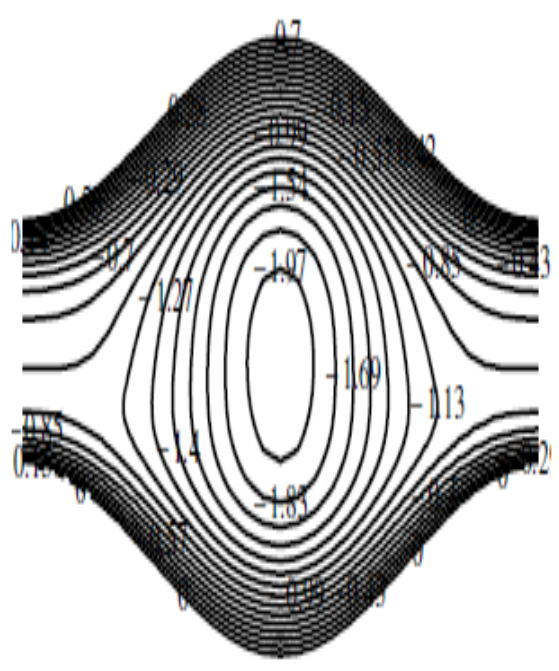

(c) $k=5$

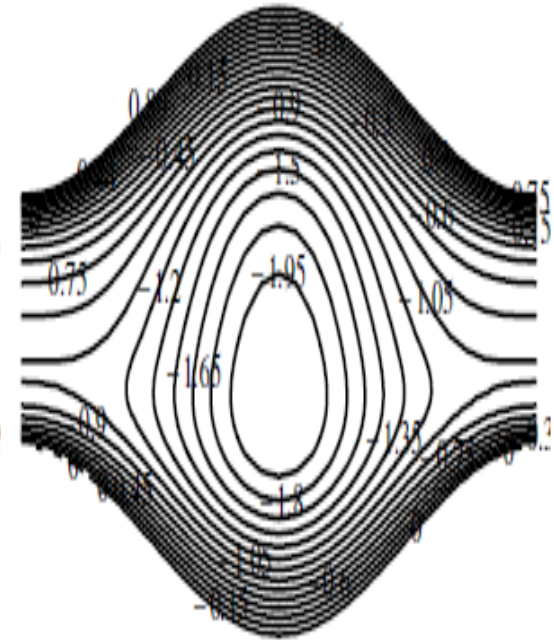

(d) $k \rightarrow \infty$

Figure 7: Nanoparticles concentration contours for channel curvature. The other parameters are $\phi=0.6, Q_{T}=1.5, B_{r}=2, U_{H S}=-1, G_{r}=1, G_{c}=1, D u=0.5, S_{r}=0.5$, $S_{c}=1, m=2, \gamma=2, R_{\zeta}=1$. 
6(a)-6(d) reveal that with similar data in Figs. 5a-d, there is only a single temperature (isothermal) structure. As the curvature parameter is enhanced this central zone is found to initially shrink, but thereafter to grow considerably (with greater stretching in the lateral direction) and exhibits a reduced proximity of isotherms. Finally, in Figs 7(a)-7(d), while a similar single zone is computed for the isosolute structure (nano-concentration contours), with increasing curvature parameter (a straighter microchannel), the zone grows consistently and there is an intensification in the proximity of the concentration contours, in particular in the lower microchannel half space. In all cases the modification in curvature exerts a significant influence on vortex structures.

\subsection{Entropy analysis}

Entropy generation is closely associated with thermodynamic irreversibility, which is encountered in all practical heat transfer processes. Different sources are responsible for entropy generation, such as heat transfer in the presence of temperature difference, the mass transfer in the presence of species concentration difference, the viscous dissipation and body forces (e.g. electrical, magnetic etc). The volumetric rate of entropy generation is defined as

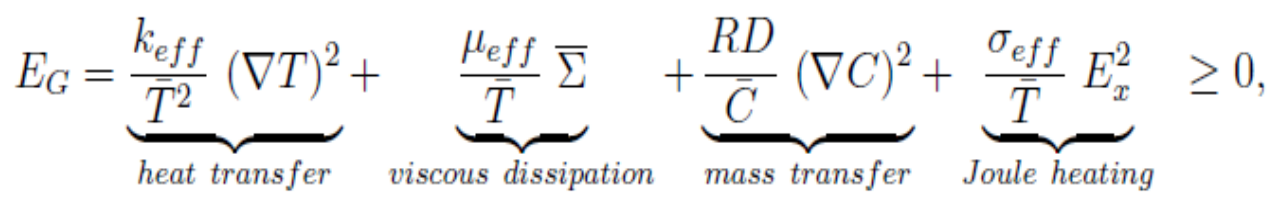

here $\bar{T}$ and $\bar{C}$ are respectively the reference temperature and concentration. The volumetric rate of a local entropy generation for incompressible Newtonian fluid in a curved channel is described in the following form as:

$$
\begin{gathered}
E_{G}=\frac{k_{e f f}}{\bar{T}^{2}}\left[\left(\frac{R}{r+R} \frac{\partial T}{\partial x}\right)^{2}+\left(\frac{\partial T}{\partial r}\right)^{2}\right]+\frac{R D}{\bar{C}}\left[\left(\frac{R}{r+R} \frac{\partial C}{\partial x}\right)^{2}+\left(\frac{\partial C}{\partial r}\right)^{2}\right] \\
+\frac{\mu_{e f f}}{\bar{T}}\left[2\left\{\left(\frac{\partial v}{\partial r}\right)^{2}+\left(\frac{R}{r+R} \frac{\partial u}{\partial x}+\frac{v}{r+R}\right)^{2}\right\}\right. \\
\left.+\left(\frac{\partial u}{\partial r}+\frac{R}{r+R} \frac{\partial v}{\partial x}-\frac{u}{r+R}\right)^{2}\right]+\frac{\sigma_{e f f}}{\bar{T}} E_{x}^{2},
\end{gathered}
$$

The dimensionless form of entropy generation rate is the entropy generation number $\left(E_{S}\right)$ which is equal to the ratio of the actual entropy generation $\left(E_{G}\right)$ to the characteristic entropy generation rate $\left(E_{C}\right)$ and is defined as

$$
E_{S}=\frac{E_{G}}{E_{C}}=\frac{a^{2} \bar{T}^{2} E_{G}}{k_{e f f}\left(T_{1}-T_{0}\right)^{2}} .
$$


Invoking the non-dimensional quantities, equation (24) can be written as

$$
\begin{aligned}
E_{S} & =\delta^{2}\left(\frac{k}{r+k} \frac{\partial \theta}{\partial x}\right)^{2}+\left(\frac{\partial \theta}{\partial r}\right)^{2}+\lambda_{1}\left[2 \delta^{2}\left\{\left(\frac{k}{r+k} \frac{\partial u}{\partial x}+\frac{v}{r+k}\right)^{2}+\left(\frac{\partial v}{\partial r}\right)^{2}\right\}\right. \\
& \left.+\left(\frac{\partial u}{\partial r}+\delta^{2} \frac{k}{r+k} \frac{\partial v}{\partial x}-\frac{u}{r+k}\right)^{2}\right]+\lambda_{2}\left[\delta^{2}\left(\frac{k}{r+k} \frac{\partial \sigma}{\partial x}\right)^{2}+\left(\frac{\partial \sigma}{\partial r}\right)^{2}\right]+\lambda_{3} .
\end{aligned}
$$

Dimensionless terms denoted $\lambda_{i}(i=1,2,3)$, are called irreversibility distribution ratios due to viscous dissipation, species concentration, and Joule heating respectively as $\lambda_{1}=\frac{\mu_{e f f} \bar{T}}{k_{\text {eff }}}\left(\frac{c}{T_{1}-T_{0}}\right)^{2}, \lambda_{2}=\frac{R D \bar{T}}{k_{\text {eff }} \bar{C}}\left(\frac{C_{1}-C_{0}}{T_{1}-T_{0}}\right)^{2}$, $\lambda_{3}=\frac{\sigma_{e f f} E_{x}^{2} \bar{T}}{k_{e f f}}\left(\frac{a}{T_{1}-T_{0}}\right)^{2}$. Figure 8 shows entropy generation contours of electroosmotic velocity $\left(U_{H S}\right)$ for curved channel $(\kappa=2)$ and for straight channel $(\kappa \rightarrow \infty)$. The entropy generation is higher in the contraction region and is comparatively lower in the expanded region of the peristaltic micro channel. This is due to the fact that the fluid friction and heat transfer rate are sufficiently large in the contracted region of the peristaltic micro channel which effectively enhances the total irreversibility. It is also observed from the figure that the entropy generation rate is higher in the inner wall and is lower in the outer wall for small values of $\kappa$. The closed contours of entropy generation rate on the centerline of the curved channel are shifted to the inner half region and entropy generation rate is large in this region (see Figs. 8(a), 8(c), 8(e)). In the case of a straight channel (see Figs. 8(b), 8(d), $8(\mathrm{f})$ ), the closed contours are on the centerline of the channel and entropy generation rate increases as one moves from the centerline of the channel to the periphery i.e. channel walls. The entropy generation rate increases at the upper half space of the expansion region in a curved channel when the electro-osmotic velocity $U_{H S}$ varies from negative values to positive value. The values of the closed contours decrease in the curved channel when the $U_{H S}$ changes from negative value to positive value. In a straight channel, the closed contour values increase when the $U_{H S}$ value changes from negative to positive. Finally, we conclude that the entropy generation rate is higher in a curved channel as compared to a straight channel and the electroosmotic body force controls the sources of entropy generation.

\section{Conclusions}

A theoretical investigation has been presented for peristaltic pumping of electro-osmotic ionic nanofluids in a curved deformable microchannel with 


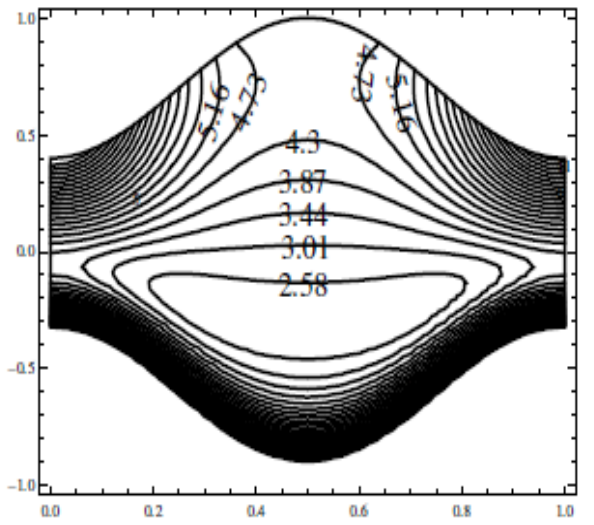

(a) $\kappa=1.5, U_{H S}=-1$

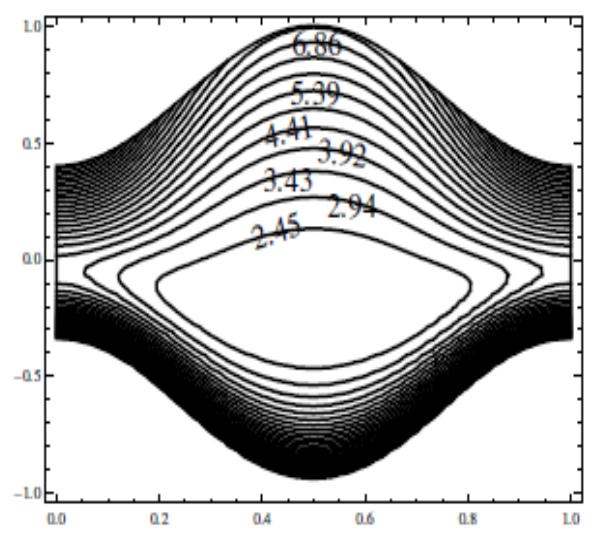

(c) $\kappa=1.5, U_{H S}=0$

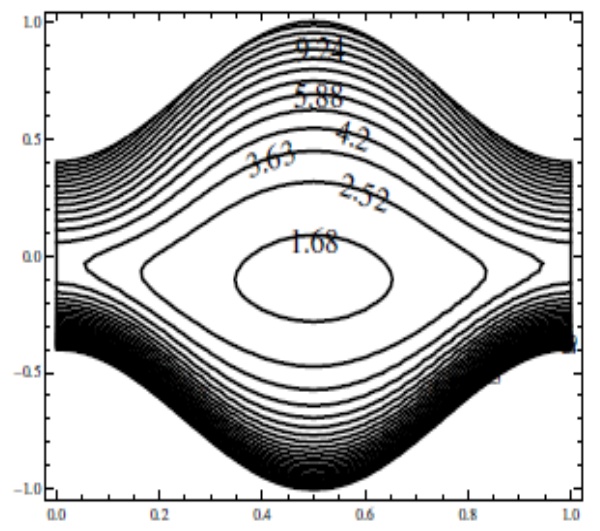

(e) $\kappa=1.5, U_{H S}=1$

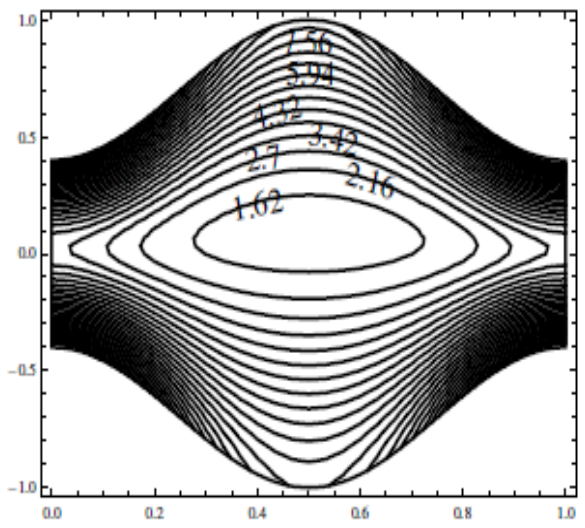

(b) $\kappa \rightarrow \infty, U_{H S}=-1$

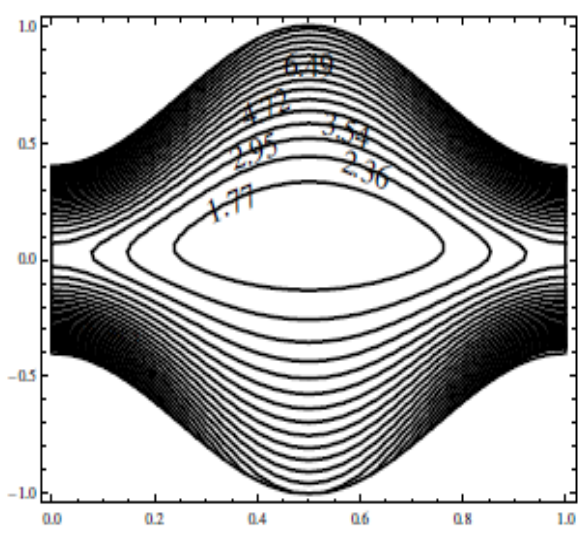

(d) $\kappa \rightarrow \infty, U_{H S}=0$

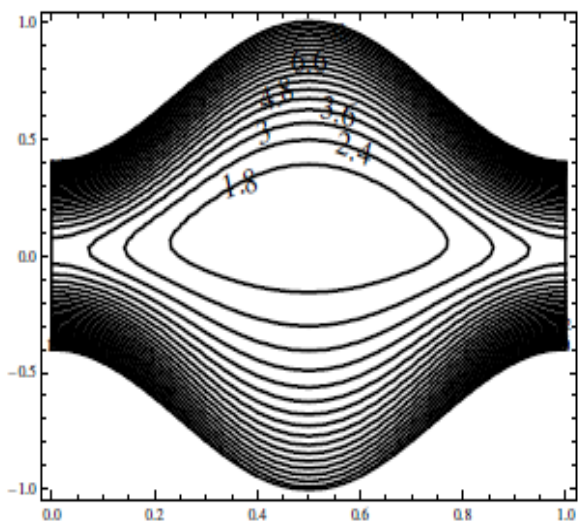

(f) $\kappa \rightarrow \infty, U_{H S}=1$

Figure 8: Entropy generation contours for different electro-osmotic velocity $U_{H S}$ with channel curvature parameter $\kappa$. The other parameters are $\phi=0.6, Q_{T}=1, B r=0.1$, $G r=1, G c=1, D u=0.5, S r=0.5, S c=1, m=1.5, \gamma=1, R_{\zeta}=1, \lambda_{1}=1, \lambda_{2}=0.5$, $\lambda_{3}=1$. 
thermal and species (nano-particle) buoyancy effects and Soret and Dufour cross-diffusion effects. Via lubrication and Debye Hückel linearization approximations, the non-dimensional conservation equations have been solved analytically. The influence of Helmholtz-Smoulochowski velocity (maximum electro-osmotic velocity), inverse EDL (electrical double layer) thickness, zeta potential ratio, curvature parameter, Brinkman number (viscous heating parameter) and Joule (electrical field heating) number on velocity, temperature, nano-particle concentration, streamline patterns, temperature contours and nanoparticles concentration contours is conducted. The computations have shown that:

1. The closed streamlines comprises two asymmetric parts in a curved channel. The magnitudes of the closed streamlines in the non-axial trapping are almost identical with increasing curvature parameter (corresponding to a progressively straight microchannel). Also increasing curvature parameter generally enhances the magnitude of closed isotherm and nanoparticle concentration contour.

2. Increasing curvature parameter induces flow deceleration in the lower micro-channel half space, whereas it generates flow acceleration in the upper micro-channel half space.

3. Increasing Brinkman number generally enhances temperatures across the micro-channel span, whereas it strongly reduces nano-particle concentration values.

4. Increasing inverse electrical double layer thickness is found to induce flow acceleration in the upper microchannel half space and flow deceleration in the lower half space, to increase temperatures and to reduce nano-particle concentration magnitudes.

5. Increasing positive Joule heating parameter increases temperatures and decreases nano-particle concentration values.

6. Increasing positive Helmholtz-Smoulochowski velocity (maximum electroosmotic velocity) decelerates the flow in the lower microchannel half space, whereas it accelerates flow in the upper microchannel half space. Increasing negative Helmholtz-Smoulochowski velocity generates the converse responses.

7. Increasing positive Helmholtz-Smoulochowski velocity (maximum electroosmotic velocity) decreases temperatures and elevates nano-particle concentration values across the microchannel span. Increasing negative Helmholtz-Smoulochowski velocity results in the opposite behavior. 
8. Entropy generation rate is a decreasing function of curvature parameter. Curved surfaces act as a strong source of entropy generation and the electroosmotic body force influences the sources of entropy generation.

\section{Appendix A.}

The vector operators in the governing equations are

$$
\begin{gathered}
(\bar{V} \cdot \nabla)=\frac{R u}{(r+R)} \frac{\partial}{\partial x}+v \frac{\partial}{\partial r}, \\
\nabla^{2}=\left(\frac{R}{r+R}\right)^{2} \frac{\partial^{2}}{\partial x^{2}}+\frac{1}{r+R} \frac{\partial}{\partial r}+\frac{\partial^{2}}{\partial r^{2}},
\end{gathered}
$$

The transformations between the wave and the laboratory frames, in dimensionless form, are:

$$
X=x-t, Y=r, U=u-1, V=v, q=F-2 h, \Psi=\psi-r,
$$

here the left hand side parameters are in the wave frame and the right hand side parameters are in the laboratory frame.

The dimensionless volume flow rate in laboratory frame is defined as

$$
F(x, t)=\int_{-h}^{h} u(x, r, t) d r,
$$

and the time averaged flow rate over one period, yielding

$$
Q_{T}(x)=\int_{0}^{1} F(x, t) d t .
$$

The relation between volume flow rate and time averaged flow rate is

$$
F(x, t)=Q_{T}+2(h(x, t)-1) .
$$




\section{Appendix B.}

The effective density and the heat capacity of the nanofluids are expressed as $\rho_{\text {eff }}=(1-\alpha) \rho_{f}+\alpha \rho_{s}$ and $\left(\rho c_{p}\right)_{e f f}=(1-\alpha)\left(\rho c_{p}\right)_{f}+\alpha\left(\rho c_{p}\right)_{s}$. Here $\alpha$ represents the nanoparticle volume fraction, the subscript $s$ refers to the solid particle phase dispersed in the nanofluid, the subscript $f$ refers to the property of a pure (base) fluid devoid of any nano-particle suspensions. For effective viscosity, the Brinkman model is considered, following Chakraborty and Roy [38] as $\mu_{e f f}=(1-\alpha) \mu_{f}+\alpha \mu_{s}$. The effective thermal conductivity can be written as $k_{\text {eff }}=k_{\text {Static }}+k_{\text {Brownian }}$. According to Hamilton-Crosser model [43], the static component can be expressed as

$$
k_{\text {Static }}=k_{f}\left[\frac{\frac{k_{s}}{k_{f}}+(n-1)\left\{1+\left(\frac{k_{s}}{k_{f}}-1\right) \alpha\right\}}{\frac{k_{s}}{k_{f}}+(n-1)-\left(\frac{k_{s}}{k_{f}}-1\right) \alpha}\right] .
$$

Where, $n$ is a shape factor to account for the differences in the shape of the particles (for spherical particles $n=3$ ). For the Brownian part, the kinetic theory of matters and Stokes' flow approximations are utilized, following Koo and Kleinstreuer [44] as

$$
k_{\text {Brownian }}=50000 \alpha \beta\left(\rho c_{p}\right)_{f} \sqrt{\frac{k_{B} T}{\rho_{s} d_{p}}} f_{1}(\alpha, T),
$$

where $d_{p}$ is the nanoparticle diameter and $k_{B}$ is Boltzmann constant. The functions $\beta$ and $f_{1}(\alpha, T)$ are defined as follows:

$$
\begin{array}{rlr}
\beta & =0.0137(10 \alpha)^{-1.6458}, \quad \alpha<1 \% \\
& =0.0011(10 \alpha)^{-1.4544}, \quad \alpha \geq 1 \%
\end{array}
$$

and

$$
\begin{aligned}
f_{1}(\alpha, T)=(-6.04 \alpha+0.4705) T & +(1722.3 \alpha-134.63), \\
& \text { for } 1 \% \leq \alpha \leq 4 \%, 300 K \leq T \leq 400 K .
\end{aligned}
$$

Another important property variation due to nano-particle suspensions is an effective enhancement in value of the electrical conductivity of nanofluids. The enhancement of electrolyte concentration in the bulk fluid elevates the electrical conductivity of the dispersion medium from its base state. This 
enhancement is due to the fact that the particles have surface groups that tend to dissociate when suspended in an aqueous solution. This results in an enhancement of total number of counterions in the system. A portion of co-ions gets expelled from the spatial domain. In order to maintain electroneutrality, an equivalent portion of counterions also leaves the domain. This augmentation of electrical conductivity is expected to alter the Joule heating to a significant extent and is expressed by Grosse et al. [45] after a rigorous mathematical analysis as:

$$
\sigma_{e f f}=\sigma_{f}+\alpha\left(f_{2}-\sigma_{s}\right),
$$

where

$$
\begin{aligned}
f_{2}=\frac{6 n_{1} e}{m d_{p}}[ & \left(\exp \left(-\frac{e \zeta}{2 k_{B} T}\right)-1\right) m^{+}-\left(\exp \left(\frac{e \zeta}{2 k_{B} T}\right)-1\right) m^{-} \\
& \left.-\frac{2}{m d_{p}} \tanh \left(\frac{e \zeta}{4 k_{B} T}\right)\left(m^{+}+m^{-}\right)\right]+\frac{3}{4 \pi d_{p}^{3}} Q_{p} m_{p},
\end{aligned}
$$

where $Q_{p}$ is the charge on each particle, $m_{p}$ is the particle electrophoretic mobility, $n_{1}$ is the equilibrium number concentration of the ions in the electrolyte solution, $d_{p}$ is the particle radius, $m^{ \pm}$are the mobilities of the positive/negative ions. The expression for $Q_{p}$ in a thin EDL limit for a symmetric 1:1 electrolyte solution, is given by

$$
Q_{p}=4 \pi d_{p}^{2} \frac{2 k_{B} T m \varepsilon_{s}}{e} \sinh \left(\frac{e \zeta}{2 k_{B} T}\right) .
$$

\section{References}

[1] A. Belkadi, A. Montillet, J. Bellettre, Biofuel emulsifier using highvelocity impinging flows and singularities in microchannels, ASME J. Energy Res. Technol. 140(1) (2017) JERT-17-1055(7).

[2] N. A. A. Qasem, S. M. Zubair, Compact and microchannel heat exchangers: A comprehensive review of air-side friction factor and heat transfer correlations, Energy Convers. Manage 173 (2018) 555-601.

[3] M. Norouzi, S. Z. Daghighi, O. A. Bég, Exact analysis of heat convection of viscoelastic fene-p fluids through isothermal slits and tubes, Meccanica 53 (2017) 817-831. 
[4] Y. Deng, Y. Zhao, W. Wang, Z. Quan, L. Wang, D. Yu, Experimental investigation of performance for the novel flat plate solar collector with micro-channel heat pipe array (mhpa-fpc), Appl. Therm. Eng. 54(2) (2013) 440-449.

[5] M. Norouzi, M. Davoodi, O. A. Bég, M. Shamshuddin, Theoretical study of oldroyd-b visco-elastic fluid flow through curved pipes with slip effects in polymer flow processing, Int. J. Appl. Comput. Math 4 (2018) 108(1-22).

[6] B. M. Fronk, K. R. Zada, Evaluation of heat and mass transfer models for sizing low-temperature kalina cycle microchannel condensers, J. Energy Resources Tech. 139(2) (2016) JERT-15-1398(10).

[7] O. A. Bég, A. Zubair, S. Kuharat, M. Babaie, Cfd simulation of turbulent convective heat transfer in rectangular mini-channels for rocket cooling applications, in: ICHTFM 2018: 20th International Conference on Heat Transfer and Fluid Mechanics.

[8] G. Hetsroni, A. Mosyak, E. Pogrebnyak, L. Yarin, Fluid flow in microchannels, Int. J. Heat Mass Transfer 48(10) (2005) 1982-1998.

[9] C. F. Chou, R. H. Austin, O. Bakajin, J. O. Tegenfeldt, J. A. Castelino, S. S. Chan, E. C. Cox, H. Craighead, N. Darnton, T. Duke, J. Han, S. Turner, Sorting biomolecules with microdevices, Electrophoresis 21 (2000) 81-90.

[10] J.-K. Chen, W.-J. Luo1, R.-J. Yang, Electroosmotic flow driven by dc and ac electric fields in curved microchannels, Jpn. J. Appl. Phys. 45(10) (2006) 7983.

[11] F. Ahmed, M. Mehrabadi, Z. Liu, G. A. Barabino, C. K. Aidun, Internal viscosity-dependent margination of red blood cells in microfluidic channels, J. Biomech Eng. 140(6) (2018) 061013(7).

[12] G. Tunc, Y. Bayazitoglu, Heat transfer in microtubes with viscous dissipation, Int. J. Heat Mass Transfer 44 (2001) 2395-2403.

[13] H. Ragueb, K. Mansouri, A numerical study of viscous dissipation effect on non-newtonian fluid flow inside elliptical duct, Energy Convers. Manage. 68 (2013) 124-132. 
[14] G. L. Morini, M. Spiga, The role of the viscous dissipation in heated microchannels, ASME J. Heat Transfer 129(3) (2006) 308-318.

[15] N. Nekoubin, Electroosmotic flow of power-law fluids in curved rectangular microchannel with high zeta, J. Non-Newtonian Fluid Mechanics 260 (2018) 54-68.

[16] I. Medina, M. Toledo, F. Méndez, O. Bautista, Pulsatile electroosmotic flow in a microchannel with asymmetric wall zeta potentials and its effect on mass transport enhancement and mixing, Chem. Eng. Sci. 184 (2018) 259-272.

[17] J. G. Santiago, Electroosmotic flows in microchannels with finite inertial and pressure forces, Anal. Chem. 73 (2001) 2353-2365.

[18] D. G. Haywood, Z. D. Harms, S. C. Jacobson, Electroosmotic flow in nanofluidic channels, Anal. Chem. 86(22) (2014) 11174-11180.

[19] P. Dutta, A. Beskok, T. C. Warburton, Electroosmotic flow control in complex microgeometries, J. Microelectromechanical Systems 11 (2002) $36-44$.

[20] M. J. Kim, A. Beskok, K. D. Kihm, Electro-osmosis-driven microchannel flows: a comparative study of microscopic particle image velocimetry measurements and numerical simulations, Exp. Fluids 33 (2002) 170-180.

[21] P. Liang, S. Wang, M. Zhao, Numerical study of rotating electroosmotic flow of oldroyd-b fluid in a microchannel with slip boundary condition, Chin. J. Phys. (2020).

[22] K. Ramesh, M. Devakar, Effect of heat transfer on the peristaltic transport of a mhd second grade fluid through a porous medium in an inclined asymmetric channel, Chin. J. Phys. 55 (2017) 825 - 844.

[23] T. S. Leu, F. C. Ma, Novel ehd-pump driven micro mixers, J. Mechanics 21 (2005) 137-144.

[24] D. Tripathi, A. Yadav, O. A. Bég, Electro-osmotic flow of couple stress fluids in a micro-channel propagated by peristalsis, Eur Phys J Plus 132 (2017) 173-185. 
[25] D. Tripathi, A. Borode, R. Jhorar, O. A. Bég, Three-layered electroosmosis modulated blood flow through a microchannel, Eur J Mech B Fluids. 72 (2018) 391-402.

[26] T. Hayat, A. Aziz, T. Muhammad, A. Alsaedi, On model for flow of burgers nanofluid with cattaneo-christov double diffusion, Chin. J. Phys. 55 (2017) 916 - 929.

[27] O. Mahian, L. Kolsi, M. Amani, P. Estellé, G. Ahmadi, C. Kleinstreuer, J. S. Marshall, M. Siavashi, R. A. Taylor, H. Niazmand, S. Wongwises, T. Hayat, A. Kolanjiyil, A. Kasaeian, I. Pop, Recent advances in modeling and simulation of nanofluid flows-part i: Fundamentals and theory, Phys. Rep. 790 (2019) 1-48.

[28] O. Mahian, L. Kolsi, M. Amani, P. Estellé, G. Ahmadi, C. Kleinstreuer, J. S. Marshall, R. A. Taylor, E. Abu-Nada, S. Rashidi, H. Niazmand, S. Wongwises, T. Hayat, A. Kasaeian, I. Pop, Recent advances in modeling and simulation of nanofluid flows-part ii: Applications, Phys. Rep. 791 (2019) $1-59$.

[29] A. T. Al-Halhouli, S. Buttgenbach, Liquid flow in curved microchannels, Int. J. Theoretical and Applied Multiscale Mechanics 1(3) (2009) 1-10.

[30] P. Bayat, P. Rezai, Microfluidic curved-channel centrifuge for solution exchange of target microparticles and their simultaneous separation from bacteria, Soft Matter 14 (2018) 5356-5363.

[31] J. Feng, G. Mingtian, X. J. Caia, X. Huaia, Viscous dissipation effect on entropy generation in curved square microchannels, Energy 36(8) (2011) 5416-5423.

[32] L. Wang, F. Liu, Forced convection in slightly curved microchannels, Int. J. Heat Mass Transfer 50 (2007) 881-896.

[33] V. Narla, D. Tripathi, Electroosmosis modulated transient blood flow in curved microvessels: Study of a mathematical model, MICROVASC RES 123 (2019) 25 - 34 .

[34] P. Goswami, P. K. Mondal, A. Datta, S. Chakraborty, Entropy generation minimization in an electroosmotic flow of non-newtonian fluid: 
effect of conjugate heat transfer, J. Heat Transfer 138 (2016) 051704(1$9)$.

[35] N. K. Ranjit, G. C. Shit, Entropy generation on electro-osmotic flow pumping by a uniform peristaltic wave under magnetic environment, Energy J. 128 (2017) 649-660.

[36] M. M. Bhatti, M. Sheikholeslami, A. Zeeshan, Entropy analysis on electro-kinetically modulated peristaltic propulsion of magnetized nanofluid flow through a microchannel, entropy 2017-19-481 (2017) 115 .

[37] J. R. Melcher, Continuum Electromechanics, MIT Press, 1981.

[38] S. Chakraborty, S. Roy, Thermally-developing electroosmotic transport of nanofluids in microchannels, Microfluid Nanofluidics 4 (2008) 501511.

[39] S. Datta, J. N. Choudhary, Effect of hydrodynamic slippage on electroosmotic flow in zeta potential patterned nanochannels, Fluid Dyn. Res. 45 (2013) 055502.

[40] B. J. Kirby, E. F. Hasselbrink, Zeta potential of microfluidic substrates. ii. data for polymers, Electrophoresis 25 (2004) 203-213.

[41] W. B. Russel, D. A. Saville, W. R. Schowalter, Colloidal Dispersions, Cambridge University Press, 1992.

[42] T. W. Ting, Y. M. Hung, N. Guo, Viscous dissipation effect on streamwise entropy generation of nanofluid flow in microchannel heat sinks, J. Energy Resources Tech. 138(5) (2016) 052002-052002-9.

[43] R. L. Hamilton, O. K. Crosser, Thermal conductivity of heterogeneous two component systems, Ind Eng. Chem. Fund. 1(3) (1962) 187-191.

[44] J. Koo, C. Kleinstreuer, Laminar nanofluid flow in micro heat sinks, Int. J. Heat Mass Transfer 48 (2005) 2652-2661.

[45] C. Grosse, S. Pedrosa, V. N. Shilov, Corrected results for the influence of size, $\zeta$ potential, and state of motion of dispersed particles on the conductivity of a colloidal suspension, J Colloid Interface Sci 265 (2003) $197-201$. 